This item was submitted to Loughborough's Research Repository by the author.

Items in Figshare are protected by copyright, with all rights reserved, unless otherwise indicated.

\title{
Reducing environmental impact of production during a rolling blackout policy - a multi-objective schedule optimisation approach
}

PLEASE CITE THE PUBLISHED VERSION

http://dx.doi.org/10.1016/j.jclepro.2015.04.038

\section{PUBLISHER}

(C) Elsevier Ltd.

\section{VERSION}

AM (Accepted Manuscript)

\section{PUBLISHER STATEMENT}

This work is made available according to the conditions of the Creative Commons Attribution-NonCommercialNoDerivatives 4.0 International (CC BY-NC-ND 4.0) licence. Full details of this licence are available at: https://creativecommons.org/licenses/by-nc-nd/4.0/

\section{LICENCE}

CC BY-NC-ND 4.0

\section{REPOSITORY RECORD}

Liu, Ying, Haibo Dong, Niels Lohse, and Sanja Petrovic. 2019. "Reducing Environmental Impact of Production During a Rolling Blackout Policy - a Multi-objective Schedule Optimisation Approach”. figshare. https://hdl.handle.net/2134/17437. 


\title{
Reducing Environmental Impact of Production during a Rolling Blackout Policy - A Multi-Objective Schedule Optimisation Approach
}

\author{
Ying Liu ${ }^{\mathrm{a}, *}$, Haibo Dong ${ }^{\mathrm{b}}$, Niels Lohse ${ }^{\mathrm{c}}$, Sanja Petrovic $^{\mathrm{d}}$ \\ ${ }^{a}$ School of Aerospace, Transportation and Manufacturing, Cranfield University, Cranfield, MK43 \\ 0AL, United Kingdom \\ ${ }^{\mathrm{b}}$ Division of Engineering, University of Nottingham Ningbo China, Ningbo, 315100, China \\ ${ }^{c}$ Wolfson School of Mechanical and Manufacturing Engineering, Loughborough University, Lough- \\ borough, LE11 3QZ, United Kingdom \\ ${ }^{\mathrm{d}}$ Business School, University of Nottingham, Nottingham, NG8 1BB, United Kingdom \\ * Corresponding author: Ying Liu, School of Aerospace, Transportation and Manufacturing, Cran- \\ field University, Cranfield, MK43 0AL, United Kingdom, \\ Email: ying.liu01@ cranfield.ac.uk, Mobil: +44(0)7400028806
}

\begin{abstract}
Many manufacturing companies in China currently are suffering from a Rolling Blackout policy for the industry electricity supply which means that the government electricity is cut off several days in every week resulting in manufacturing companies illegally starting their own diesel generators to maintain production. However, the private generation of electricity is more polluting and costly than the government supplied resource. Thus, the increased price of energy and the requirement to become more environmentally sustainable exert substantial pressures on manufacturing enterprises to reduce energy consumption for cost saving and to become more environmentally friendly. Scheduling of less energy consumption critical operations during rolling blackout periods can help minimise the negative effect of this policy. This is a multi-objective optimisation problem as production due dates cannot be ignored and cost is not directly proportional to electricity consumption anymore. Optimal scheduling even of relatively small production orders is clearly beyond the capability of manual tools or common single objective scheduling optimisation methods. Therefore, a multi-objective scheduling optimisation method has been developed which includes reducing electricity consumption and its related cost as part of the objectives in addition to total weighted tardiness. This research focuses on classical job shop environments which are widely used in the manufacturing industry in China and the rest of the world. A mathematical model for the triobjectives problem that minimises total electricity cost, total electricity consumption and total weighted tardiness has been developed. A specific heuristic has been devised for investigating how the Rolling Blackout policy affects the performance of existing scheduling plans. This heuristic can also be used as a remedial measurement by plant managers
\end{abstract}


if they do not have access to multi-objective optimisation tools. The Non-dominant Sorting Genetic Algorithm has been used as the basis for solving the optimisation problem. Case studies based on four modified job shop instances have been studied to show the effectiveness of the proposed heuristic and the algorithm.

Keywords: Energy efficient production planning; Sustainable manufacturing; Job shop scheduling

\section{Introduction}

The manufacturing industry is one of the most important energy consumers and carbon emitters in the world. For instance, every year in China, manufacturing consumes around 50\% of the entire electricity produced (Tang et al., 2006), and generates at least $26 \%$ of the total carbon dioxide emissions. In order to reduce the carbon emission and balance the time-based unevenness of electricity demand, a Rolling Blackout policy is promulgated to industry electricity supply in some areas of China. This means the government electricity is cut off several days in every week for a specific company. This policy creates huge difficulties to manufacturing companies since their production can be significantly limited by the resulting lack of electricity to power their production machines. For some companies, up to $1 / 3$ of their production capacity can be lost. To recover their production capacity, some companies illegally start their own diesel generators. This increases their electricity costs as the cost of this privately generated electricity is twice as high as the government supplied resource. The original intention of implementing the Rolling Blackout policy is to reduce emissions. Ironically, it results in the wide generation of private electricity which is more emission intensive. Based on the above discussion and the background of increasing price of energy (Kilian, 2008), a new objective for many manufacturing companies in China is to reduce electricity consumption and cost during production as well as ensure quality and customer satisfaction when the Rolling Blackout policy is implemented.

The challenge they face is that as a result of the Rolling Blackout policy, minimising electricity consumption and cost while maintaining their order due dates, has become a none trivial triple objective optimal scheduling problem. Finding even close to optimal solutions is much beyond the capability of commonly used single objective methods which only consider the time domain. Furthermore, specific heuristics to consider the implications of the Rolling Blackout during scheduling have not yet been investigated. Hence, there is a clear need to first of all understand the potential of applying operational research methods to solve this problem. Secondly, simple heuristics should be defined to 
aid practitioners with improving the optimality of their schedules even without lengthy multiobjective optimisation which is often beyond the capabilities and skill sets of average small to medium sized companies.

Previous research by (Mouzon \& Yildirim, 2008; Shrouf et al., 2014) confirm that operational research methods are providing an effective approach to reduce the energy consumption and its related cost to manufacturing companies by improving their production schedules. This is especially true in the mass production environments as production schedules are less complex yet any improvement can have a large effect due to large production volumes (Gutowski et al., 2005). Job shop type manufacturing environment are far more common but also inherently more complex due to larger product variety and diversity resulting in more complex material handling requirements. Consequently, optimising schedules for such environments is more challenging especially if multiple objectives need to be considered.

A very large number of small to medium sized companies in China operate a job shop production environment. Hence, the job shop has been selected as focus of this research. A general model of the tri-objective job shop scheduling problem that consider minimising total electricity cost (TEC), total non-processing electricity consumption (NPE) and total weighted tardiness (TWT) when the Rolling Blackout policy is applied (Electricity Consumption, Electricity Cost and Tardiness-EC2T) has been proposed in this paper for the first time. To solve this problem, the influence that the Rolling Blackout policy exerts on the existing scheduling plans of manufacturing companies needs to be understood first. To achieve this, a new heuristic has been developed to adjust existing scheduling plans to fit the government electricity supply time frame when the Rolling Blackout policy has been applied. This heuristic can also be used as a remedial measure for plant managers which enable them to minimise their TWT deterioration. A comparison between the adjusted and original schedules can demonstrate the policy's influence on TWT. In the adjustment scenario, the use of private electricity is forbidden to illustrate the worst case effect on schedules. Comparatively, the other solution for the manufacturing companies is to use the private electricity to maintain the existing scheduling plans when the policy is applied. It can be expected that the electricity cost will increase in this scenario since the private resource is more expensive. Based on the aforementioned two scenarios, it can be expected that, at least one of the schedule's performance indicator will be adversely affected (TWT or TEC), whether private electricity is used or not during the government electricity unavailable periods. This is the motivation for developing compromised scheduling plans to realise the trade-off between TWT and TEC. To solve this problem, the Non-dominant Sorting Genetic Algorithm 
(NSGA-II) (Deb et al., 2002) has been adapted for achieving the tri-objective optimisation of the EC2T problem with new encoding method for the first time.

In the remainder of the paper, following the background and research motivation in Section 2, the mathematical model of the EC2T is presented in Section 3. Then the procedure of the developed adjustment heuristic is presented in Section 4. A scheduling plan comparison experiment is presented in this section to investigate the influence that the Rolling Blackout policy exerts on existing schedules. In Section 5, a new encoding schema as well as crossover and mutation operators are provided to solve the EC2T and a case study is presented to demonstrate the effectiveness of the algorithm for the research problem.

\section{Background and motivation}

The amount of reported research in the area of scheduling with environmentally-oriented objectives is currently small but increasing. The most relevant studies were conducted by Mouzon (2008) and He et al. (2012). Based on their work and the approach developed by Kordonowy (2003) which individually considers the total energy used by each stage of machining processes, Liu et al. (2014) have further divided the electricity consumption of each machine tool into two components: the nonprocessing electricity consumption (NPE) and processing electricity consumption (PE) for scheduling optimisation. NPE is associated with machine start-up, shut-down and idling. The electricity consumed when a job is processed on a specific machine can be defined as job related processing electricity consumption (JPE), including the basic power consumption of machine tools, i.e. idle power, the runtime operations and the actual cutting consumption. Thus, PE is the sum of all the JPE on a specific machine. The JPE for the processing of each job can be considered to be a constant in a given manufacturing system provided that there are only single or the same type of machines for each operation. In this case, it has been proved by Liu et al. (2014) that by adjusting scheduling plans in a basic job shop only the total NPE can be reduced.

Based on the above, the current electricity saving methods (ESMs) at the manufacturing system level include: Sequencing, Turn Off/Turn On and Process Route Selection (PRS). The Sequencing method reduces the non-processing time of the machines in the system by changing the order of jobs on a machine. This reduces the idle electricity consumption of the system. The Turn Off/Turn On method (Mouzon, 2008) looks for opportunities to save electricity by turning off a machine tool when it becomes idle for a sufficient period of time. The Sequencing and Turn Off/Turn On can be applied to any type of manufacturing system to reduce the NPE provided that the start-up and shut-down times 
and energy requirements of the machines do not exceed the opportunities for saving. Comparatively, PRS is only applicable to workshops with alternative routes. It can reduce both PE and NPE by choosing different processing routes for job. Its ineffectiveness in workshops without alternative routes or having identical alternative routes for jobs, for instance, the job shop environment limits its range of application. Dispatching rules, a genetic algorithm and a greedy randomised adaptive search procedure have been proposed by Mouzon et al. (2007) and Mouzon (2008) to optimally use the aforementioned three methods to reduce both total NPE and PE for single machine and parallel machine environments. He et al. (2012) used PRS to decrease both total PE and total NPE for a flexible job shop environment. In addition, Bruzzonea et al. (2012) developed a method to modify the schedule of the jobs in flexible flow shops in order to adjust to the maximum peak power constraint. Subai et al. (2006) consider energy and waste reduction in hoist scheduling problem of the surface treatment processes without changing the original productivity. Research considering the electricity price pattern as constraint, thereby achieving the cost saving is scarce. Herrmann and Thiede (2009) considered the use of operational research methods to reduce the total energy cost when manufacturing plants are charged based on the peak power demand from the energy provider instead of the actual electricity consumption. Fang et al. (2011) considered reducing peak power load in a flow shop. Gupta and Venkataraman (2013) developed a framework to assess strategies for optimally shifting peak load electricity consumption using distributed storage systems. The objective is to reduce the price risk because of fluctuating demand for electricity. Focusing on avoiding peak times and considering variable energy prices, Luo et al. (2013) addressed a new ant colony optimisation to solve hybrid flow shop scheduling by considering production-efficient electricity consumption. They proposed a multi-objective solution for minimising the makespan and energy consumption power with time-of-use prices. Kuster et al. (2013) use evolutionary algorithms and multi-agent technology to reconfigure production schedule, to reduce energy costs through utilising periods with low energy prices. This is enabled by shifting high energy consuming parts to the low energy price periods.

Based on the discussion above, it can be found that employing operational research methods to reduce the total electricity cost, total electricity consumption as well as keeping good performance on traditional job shop scheduling indicator in a typical job shop environment when the Rolling Blackout policy is applied still has not been explored. In addition, the influence that the Rolling Blackout policy exerts on the existing scheduling plans has not been investigated. 


\section{Problem definition and notations}

The notations used in the problem statement, algorithm description and throughout the paper are as follows:

\begin{tabular}{|c|c|}
\hline$B_{k}$ & $\begin{array}{l}\text { the break-even duration of machine } M_{k} \text { for which Turn } \\
\text { Off/Turn On is economically justifiable instead of running the } \\
\text { machine idle }\end{array}$ \\
\hline$C_{i}(s)$ & $\begin{array}{l}\text { completion time of } J_{i} \text { in schedule } s \text { (i.e. the completion time of } \\
\text { the last operation of } J_{i}, O_{i}^{u_{i}} \text { ) }\end{array}$ \\
\hline$C_{k}^{r}$ & completion time of $m_{k}^{r}$ on $M_{k}$ \\
\hline$d_{i}$ & due date of $J_{i}$ \\
\hline$E_{k}^{\text {turn }}$ & electricity consumed by Turn Off/Turn On \\
\hline$f$ & tardiness factor \\
\hline$J$ & a finite set of $n$ jobs, $J=\left\{J_{i}\right\}_{i=1}^{n}$ \\
\hline$M$ & a finite set of $m$ machines, $M=\left\{M_{k}\right\}_{k=1}^{m}$ \\
\hline$M_{k}^{\prime}$ & $\begin{array}{l}\text { a finite set of operations processed on } M_{k} \\
M_{k}^{\prime}=\left\{m_{k}^{r}\right\}_{r=1}^{\sum_{i=1}^{n} \Sigma_{l=1}^{u_{i}} \gamma_{i k}^{l}}\end{array}$ \\
\hline $\begin{array}{c}m_{k}^{r} \\
m_{k}^{r-1}\end{array}$ & $\begin{array}{l}r \text {-th operation processed on } M_{k} \text { within a feasible schedule } s \\
m_{k}^{r} \text {, s preceding operation on the same machine } M_{k}\end{array}$ \\
\hline$O_{i}$ & a finite set of $u_{i}$ ordered operations of $J_{i}, O_{i}=\left\{O_{i k}^{l}\right\}_{l=1}^{u_{i}}$ \\
\hline $\begin{array}{l}O_{i k}^{l} \\
p_{i k}^{l}\end{array}$ & $\begin{array}{l}l \text {-th operation of } J_{i} \text { processed on } M_{k} \\
\text { processing time of } O_{i k}^{l}\end{array}$ \\
\hline$P_{k}(t)$ & input power of $M_{k}$ \\
\hline$P_{k}^{i d l e}$ & idle power of $M_{k}$ \\
\hline$r_{i}$ & release time of $J_{i}$ into the system \\
\hline $\begin{array}{l}\gamma_{i k}^{l} \\
S\end{array}$ & $\begin{array}{l}\gamma_{i k}^{l}=1 \text { if the } l \text {-th operation of } J_{i} \text { processed on } M_{k}, 0 \text { otherwise } \\
\text { a feasible schedule plan }\end{array}$ \\
\hline$S$ & a finite set of all feasible schedule plans, $s \in S$ \\
\hline$S_{k}^{r}$ & starting time of $m_{k}^{r}$ on $M_{k}$ \\
\hline$T_{i}(s)$ & $\operatorname{tardiness}$ of $J_{i}$, defined as $T_{i}(s)=\max \left\{0, C_{i}(s)-d_{i}\right\}$ \\
\hline$T$ & the cycle period of the Rolling Blackout policy \\
\hline$t_{s}$ & the time point which separates $T$ from $\Delta t_{s}$ and $\Delta t_{o}$ \\
\hline$\Delta t_{s}$ & government electricity supply available period, GAP \\
\hline$\Delta t_{o}$ & government electricity supply unavailable period, GUP \\
\hline$t_{k}^{O F F}$ & time required to turn off $M_{k}$ and turn on it back on \\
\hline$w_{i}$ & weighted importance of $J_{i}$ \\
\hline $\begin{array}{l}Y_{i i^{\prime} k}^{l l^{\prime}} \\
Z_{k}^{r}\end{array}$ & $\begin{array}{l}Y_{i i^{\prime} k}^{l l^{\prime}}=1 \text { if } O_{i k}^{l} \text { precedes } O_{i^{\prime} k}^{l^{\prime}} \text { on } M_{k}, 0 \text { otherwise } \\
Z_{k}^{r}=1 \text { if } S_{k}^{r+1}-C_{k}^{r} \geq \max \left(B_{k}, t_{k}^{O F F}\right), 0 \text { otherwise }\end{array}$ \\
\hline
\end{tabular}


The tri-objective EC2T problem is developed based on the bi-objective ECT problem which has been formally defined by Liu et al. (2014) based on the work of Özgüven et al. (2010). A new objective which is to minimise the electricity cost when the Rolling Blackout policy is applied has been added. The first part of the EC2T model describes the classical job shop scheduling problem where a finite set of $n$ jobs $J=\left\{J_{i}\right\}_{i=1}^{n}$ are to be processed on a finite set of $m$ machines $M=\left\{M_{k}\right\}_{k=1}^{m}$ following a predefined order. Each job is defined as a finite set of $u_{i}$ ordered operations $O_{i}=\left\{O_{i k}^{l}\right\}_{l=1}^{u_{i}}$ where $O_{i k}^{l}$ is the $l$-th operation of job $J_{i}$ processed on machine $M_{k}$ and requiring a processing time $p_{i k}^{l}$. The time that each job $J_{i}$ is released into the system is denoted by $r_{i}$ and the due date of the job is defined by $d_{i}$. Different jobs can be prioritised using the weighted importance factor $w_{i}$. Given a feasible schedule $s$, let $C_{i}(s)$ indicate the completion time of job $J_{i}$ in that schedule. The tardiness of job $J_{i}$ can then be denoted as $T_{i}(s)=\max \left\{0, C_{i}(s)-d_{i}\right\}$. The first optimisation objective is to minimise the total weighted tardiness of all jobs, which can be expressed as:

$$
\operatorname{minimise}\left(\sum_{i=1}^{n} w_{i} \times T_{i}(s)\right)
$$

The second part of the EC2T model depicts the electricity consumption of the machine tools in a job shop. A simplified mathematical power input model for a machine $M_{k}$ when it is working on operation $O_{i k}^{l}$ has been developed by Liu et al. (2014). There are three constant levels of power consumption for each machine: during idle time, when switched into run-time mode and when carrying out the actual cutting operation. A stepped function represented by the red line in Figure $\mathbf{1}$ denotes the input power $P_{k}(t)$ a machine requires over time. The idle power level of a machine is defined by $P_{k}^{i d l e}$. The overall processing time $p_{i k}^{l}$ is defined as the time interval between coolant switching on and off. The objective to reduce the total electricity consumption in a job shop is realised by reducing the total NPE. Hence, the objective function which minimises the total non-processing electricity consumption in a job shop to carry out a given job schedule can be expressed as:

$$
\operatorname{minimise}\left(\sum_{k=1}^{m} T E M_{k}^{n p}(s)\right)
$$

Where $T E M_{k}^{n p}(s)$ represents the NPE of machine $M_{k}$ for schedule $s$. The NPE is a function of the scheduling plan which needs to be expressed based on the specific order that all operations have been scheduled to run on a machine. $M_{k}^{\prime}=\left\{m_{k}^{r}\right\}_{r=1}^{\sum_{i=1}^{n} \Sigma_{l=1}^{u_{i}} \gamma_{i k}^{l}}$ is the finite set of operations processed on $M_{k}$; $\gamma_{i k}^{l}$ is a decision variable that $\gamma_{i k}^{l}=1$ if the $l$-th operation of job $J_{i}$ processed on $M_{k}, 0$ otherwise; $S_{k}^{r}$ and $C_{k}^{r}$ respectively indicate the start and completion time of operation $m_{k}^{r}$ on $M_{k}$ for a schedule $s$. Since the Turn Off/Turn On operation is applied to idle periods which are long enough, the idle elec- 
tricity consumption by these periods are replaced by the electricity consumed by turning the machine off and then turning it back on. According to Mouzon et al. (2007), $E_{k}^{\text {turn }}$ is the electricity consumed by Turn Off/Turn On. $B_{k}$ is the break-even duration of machine $M_{k}$ for which Turn Off/Turn On is economically justifiable instead of running the machine idle, $B_{k}=E_{k}^{\text {turn }} / P_{k}^{\text {idle }}$. A Turn Off/Turn On can be applied to an idle period if it is longer than or equal to the maximum value between breakeven duration of $M_{k}$ and the time required to turn off $M_{k}$ and turn it back on. $Z_{k}^{r}$ is a decision variable that $Z_{k}^{r}=1$ if a Turn Off/Turn On is applied to the idle period following $C_{k}^{r}, 0$ otherwise. Consequently, the calculation of the NPE of machine $M_{k}$ can be expressed as:

$$
T E M_{k}^{n p}(s)=P_{k}^{i d l e} \times\left[\max \left(C_{k}^{r}\right)-\min \left(S_{k}^{r}\right)-\sum_{r}\left(C_{k}^{r}-S_{k}^{r}\right)-\sum_{r}\left(S_{k}^{r+1}-C_{k}^{r}\right) \times Z_{k}^{r}\right]+E_{k}^{\text {turn }} \times \sum_{r} Z_{k}^{r}
$$

To obtain the value of NPE of a specific machine by Equation (3), firstly the total idle time of that machine needs to be calculated. Then, the idle periods that the Turn off/Turn on operation has been applied should be subtracted from the total idle time. Then, the aforementioned value is multiplied by the idle power level of the machine to obtain the total idle electricity consumption. Finally, the electricity consumed by the Turn off/Turn on operation should be summed with the idle electricity consumption to get the value of NPE. An example for the calculation of the NPE of a machine is presented by Liu (2014).

The final part of the EC2T model describes the electricity cost in a job shop when the Rolling Blackout policy is applied. It is difficult to estimate the loss for manufacturing companies during the periods when no electricity is available from the public supplier (government electricity unavailable period, GUP). To simplify it, it can be supposed that manufacturing companies can start the private power supplement with a higher cost for it. Thus, the loss during the electricity unavailable periods can be totally converted to increasing electricity cost. This can simplify the calculation for cost. In reality, the Rolling Black policy would be executed as cutting off the government electricity supply several days in every week. The policy has been abstracted as mathematical model as seen below. The objective function for electricity cost of a job shop based on the Rolling Blackout policy is:

$$
\begin{gathered}
\operatorname{minimise}(\operatorname{TEC}(s)) \\
\operatorname{TEC}=\sum_{k=1}^{m} \operatorname{TE} C_{k}(s) \\
\operatorname{TEC}_{k}(s)=p^{e} \times \int_{0}^{\max \left(C_{k}^{r}\right)} P_{k} \quad p^{e}= \begin{cases}\beta_{1}, & t \in\left[(n-1) T,(n-1) T+t_{s}\right] \\
\beta_{2}, & t \in\left((n-1) T+t_{s}, n T\right]\end{cases}
\end{gathered}
$$


$T E C(s)$ and $T E C_{k}(s)$ refer to the total electricity cost of a job shop and a machine in a feasible schedule $s$, respectively. $p^{e}$ represents the electricity price such that $p^{e}=\beta_{1} M U s / k W h$ if it is government electricity supply, and $p^{e}=\beta_{2} M U s / k W h$ if it is private electricity supply such as diesel. MU means money unit. As seen in Figure 1, $T$ denotes the cycle period of the Rolling Blackout policy. $t_{s}$ is the time point which separates $T$ from $\Delta t_{s}$ and $\Delta t_{o}$ which respectively indicate the periods with (Government electricity supply available period, GAP) and without the government electricity supply (GUP). $n$ is the natural number starting from 1 ; and $t$ indicates the time.

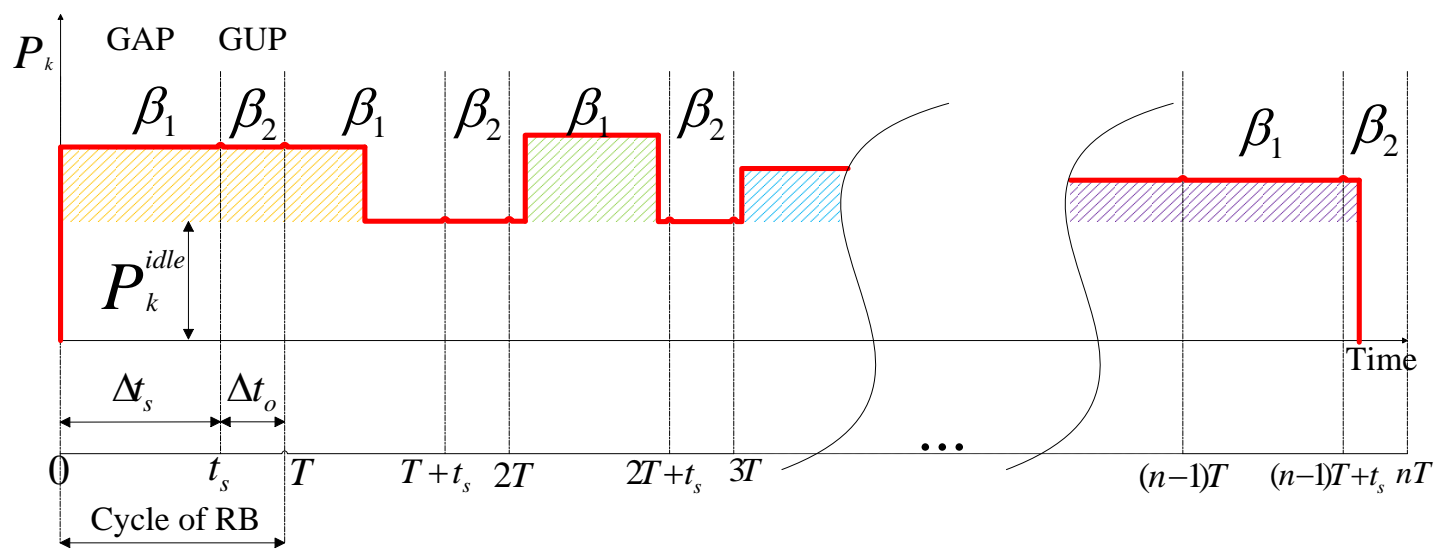

Figure 1 The timeline for the RB and the power input profile of $M_{k}$

In summary, the three objective function for minimising TWT, total NPE and TEC can be expressed as Equations (7) and (8):

$$
\begin{gathered}
\text { minimise } F(s)=\left(f_{1}(s), f_{2}(s), f_{3}(s)\right) s \in S \\
f_{1}(s)=\sum_{i=1}^{n} w_{i} \times T_{i}(s) ; f_{2}(s)=\sum_{k=1}^{m} \operatorname{TEM}_{k}^{n p}(s) ; f_{3}(s)=\operatorname{TEC}(s)
\end{gathered}
$$

\section{Investigation of the Rolling Blackout policy's influence on job shops}

The aim of this section is to demonstrate how the Rolling Blackout policy affects the performance of the scheduling plans in terms of total weighted tardiness, total electricity cost and total nonprocessing electricity consumption. The objective function values of three types of schedule are compared, including: the original schedules (OS), the adjusted schedules (AS) and original schedules with private electricity (OSPE). The OS are produced by a new multi-objective genetic algorithm (Liu, 2014). Both the Turn off/Turn On and Sequencing methods are used in this optimisation procedure. By applying the Rolling Blackout policy and the developed adjustment heuristic on OS, the AS can be obtained. OSPE represents the situation that the private electricity is provided during all the electricity supply unavailable periods. Thus, the original schedule is not changed. The total electrici- 
ty cost can be obtained by applying different electricity prices for electricity supply available periods and electricity supply unavailable periods. The objective function values of the aforementioned three types of schedules are compared to demonstrate the influence that the Rolling Blackout police exerts on existing scheduling plans. The procedure of the adjustment heuristic is introduced in the following sections.

\subsection{The procedure of the adjustment heuristic}

The idea behind the developed adjustment heuristic is that when a Rolling Blackout policy is being applied, the operations locate in the government electricity unavailable period and their subsequent operations on the same machine and in the same job first need to be postponed. As shown in Figure 2, the period between 450 and 600 time units is the first government electricity unavailable period. Thus, all operations after the red line which indicates the first splitting line of the schedule need to be moved right. The sequence and pattern of these operations are kept the same after the right move. Applying the right move is based on the fact that the original scheduling plan is an optimal one, thus it is beneficial to keep its sequence and pattern. After this right move, a new schedule can be obtained with no operations locates in the time period between 450 and 600 time units. Then, the operations locate in the second government electricity unavailable period, which is between 1050 and 1200 and their subsequent operations on the same machine and in the same job need to be postponed. One should notice that the operations which are located in the time period between 1050 and 1200 time units in the above step are not those shown in Figure 2. They are the same operations which had been postponed to this time range after the first right move. After finishing all the right moves, it might be found that some of the operations can be moved left to improve the schedule's performance on the total weighted tardiness. The adjusted schedules can be obtained after finishing the left moves. 


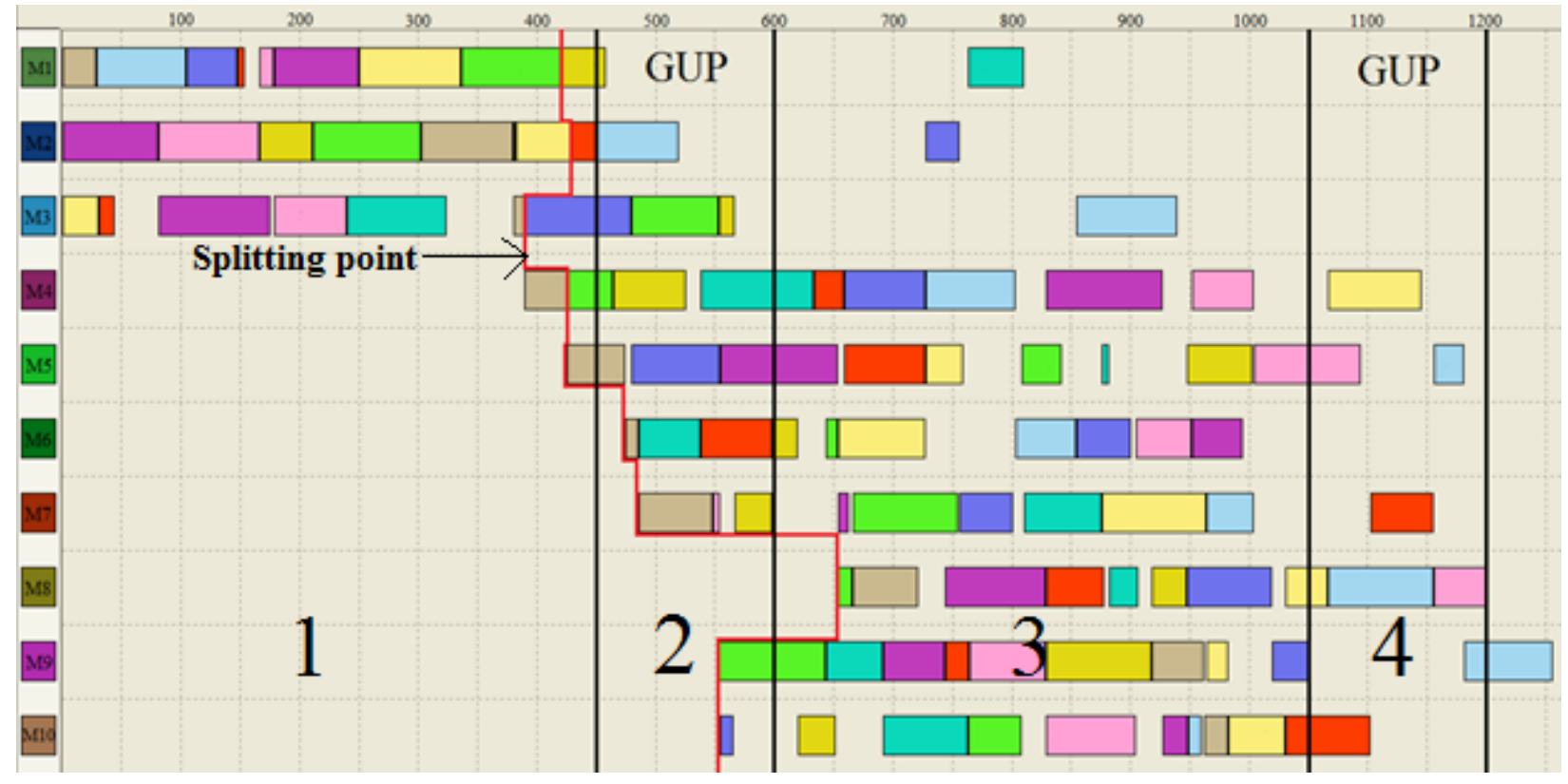

Figure 2 Example for right move

The flowchart of the adjustment heuristic is presented in Figure 3. The process of the heuristic has been described in detail in the following section. Based on the aforementioned information, all the government electricity supply available periods (GAPs) and the government electricity supply unavailable period (GUPs) in a schedule $s$ can be enumerated. The GAPs are the odd periods which can be numbered as $2 x-1$ th period and GUPs are the even periods which can be numbered as $2 x$ th period, where $x=1,2,3 \ldots a$. As seen in Figure 2, the 1 th and 3 th periods are the GAPs, while the 2 th and 4 th periods are the GUPs. 


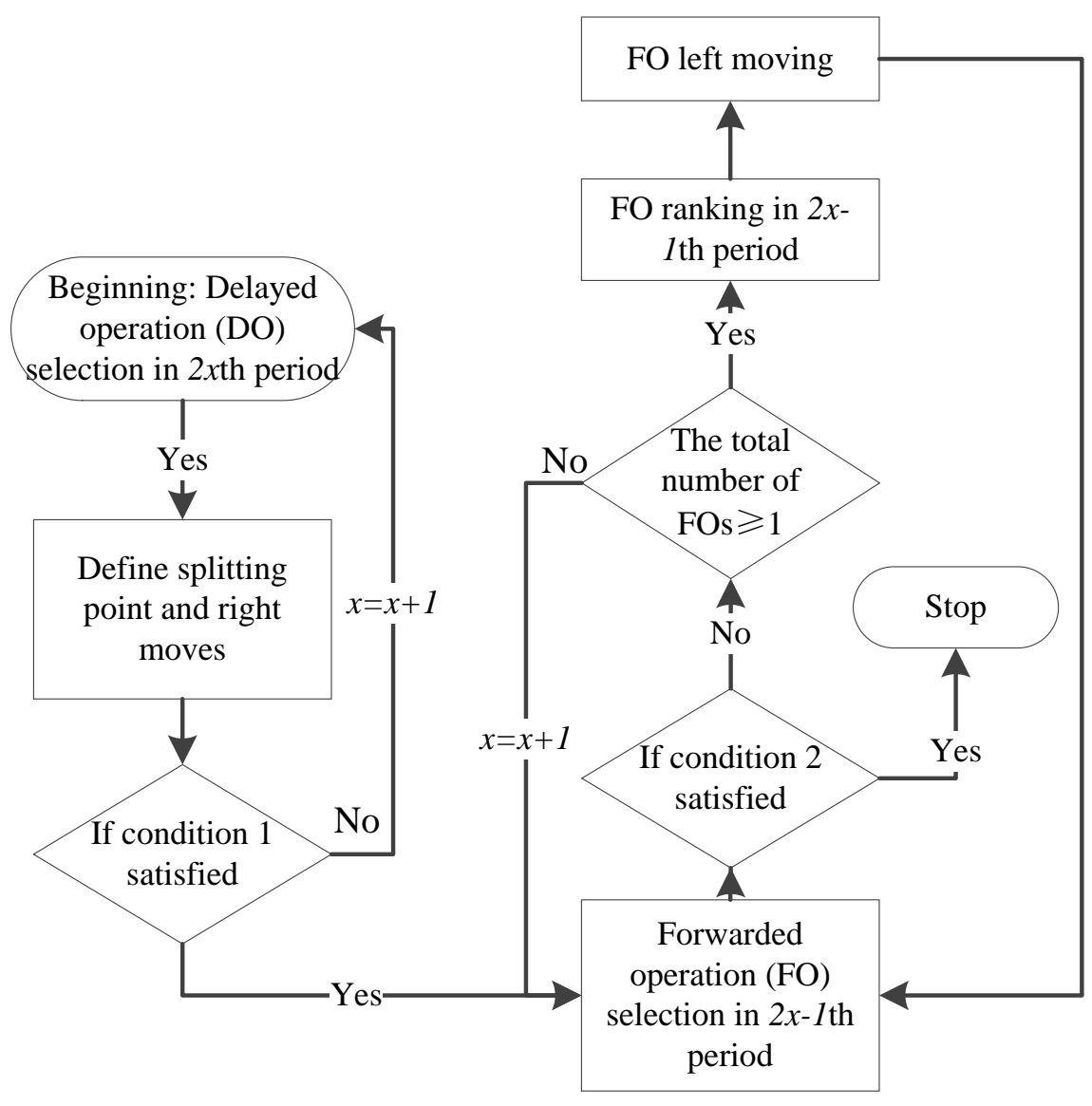

Figure 3 the flowchart of the adjustment heuristic

Delayed operation selection: The task in this step is to find out operations which need to be delayed due to unavailable electricity supply in GUP periods, which are the even periods shown in Figure 2. They can be denoted as $2 x$ th periods. The search starts from period 2. $O_{i k}^{l}$ can be defined as delayed operation related to the $2 x$ th period if any part of its processing time locates in the $2 x$ th period.

Define splitting point and right moves: after identifying all the delayed operations related to the $2 x$ th periods, the splitting line and splitting point on schedule $s$ needs to be defined. The starting times of all the delayed operations form the splitting line. See the red line in Figure 2 showing the split line for the first GUP period. All operations after the splitting line need to be postponed. The earliest starting time of all the delayed operations is defined as the splitting point. The new value for this point equals to the starting time of the government electricity supply available period (GAPs, which are the odd ones denoted by period $2 x+1$ ) following the $2 x$ th period. In the case presented in Figure 2, the new value for the splitting point is the 600 time unit. 


\section{Condition 1:}

Condition 1 is used to judge whether all operations that need to be postponed have finished their right move. If all the last operations on every machine are finished before the starting time of the $2 x$ th period, which means all of the operations have been moved to the government electricity supply available periods, the algorithm goes to the next step which is the forwarded operation selection step. Otherwise, the delayed operation selection related to the $2(x+1)$ th period is executed. A schedule which finishes all right moves is presented in Figure 4, denoted as $s^{\prime}$.

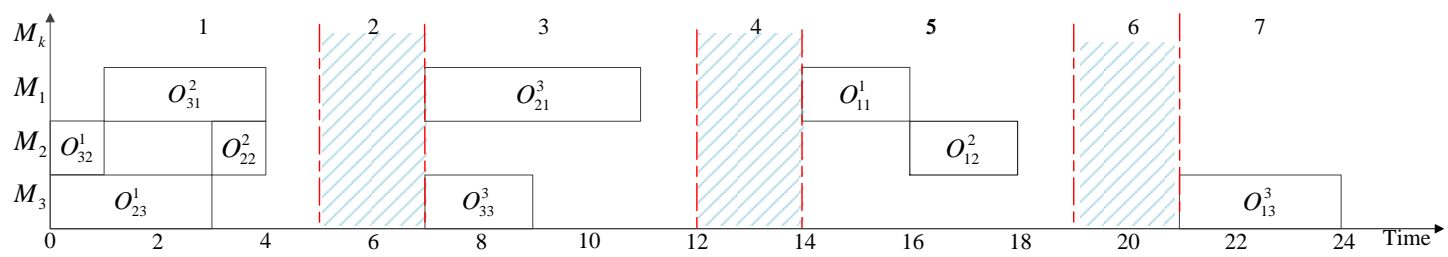

Figure 4 A scheduling plan finished all the right moves

Forwarded operations selection: In some cases, it can be noticed that some of the operations can be moved back forward (left) after finishing all the right moves. Thus, the schedule's total weighted tardiness performance can be improved. Therefore, operations in $2 x-1$ th periods (GAPs) within the schedule $s^{\prime}$ which can be moved forwarded should be identified and moved left. The search starts from the 1 th period, and move to the $2(x+1)-1$ th period to begin a new search procedure if there is no forwarded operation related to the $2 x-1$ th period. An operation can be defined as forwarded operation in the $2 x-1$ th period if its position on the scheduling plan looks like the target operation $O_{i k}^{l}$ in Figure 5-Figure 7. Within the three figures, POJ is defined as operation $O_{i k}^{l}$,s preceding operation within the same job. Similarly, POM is operation $O_{i k}^{l}$ 's preceding operation on the same machine.

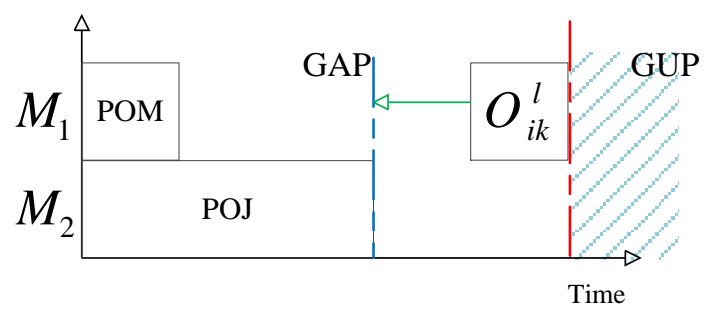

Figure 5 Feasible forwarded operation (in one GAP) 


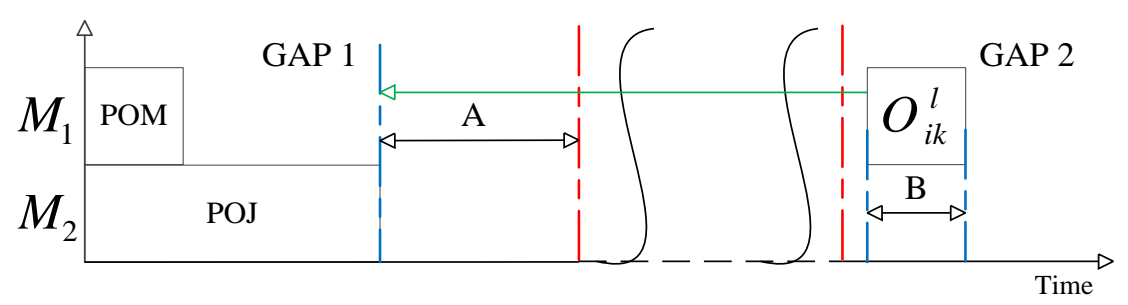

Figure 6 Feasible forwarded operation (in more than one GAP) situation 1

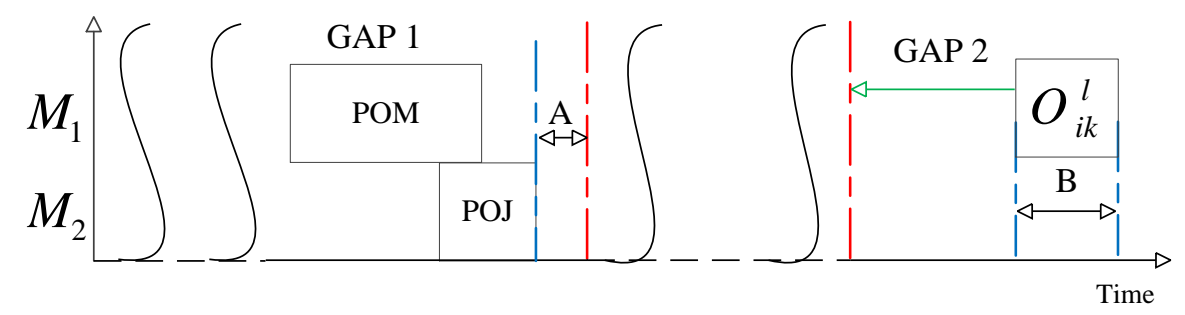

Figure 7 Feasible forwarded operation (in more than one GAP) situation 2

As shown in Figure 5, when the starting time of operation $O_{i k}^{l}(\mathrm{SO})$ and the maximum value between the completion time of $O_{i k}^{l}$ 's preceding operation within the same job (CPOJ) and the completion time of $O_{i k}^{l}$ 's preceding operation on the same machine (CPOM) are in the same government electricity supply available period, $O_{i k}^{l}$ can be defined as a forwarded operation if SO is larger than the maximum between CPOJ and CPOM. $O_{i k}^{l}$ can then be moved left to the blue dash line which is the maximum between CPOJ and CPOM. When SO and the maximum between CPOJ and CPOM belong to different government electricity supply available periods, $O_{i k}^{l}$ can be defined as a forwarded operation if the processing time of $O_{i k}^{l}$ is smaller than the gap between the ending time of the period where the maximum between CPOJ and CPOM locates and the CPOJ or CPOM itself, as shown in Figure 6. $O_{i k}^{l}$ can then be moved left to the blue dash line which means the new starting time of the operation equals to the maximum between CPOJ and CPOM. The final situation is shown in Figure 7 which is similar to the situation shown in Figure 6, but the space left in the period where the maximum between CPOJ and CPOM locates is not enough for $O_{i k}^{l}$ to move into. Then the target operation can be moved to the starting time of the first government electricity supply available period following the GAP where the maximum between CPOJ and CPOM locates, if the target operation originally started later than that time point.

\section{Condition 2:}

Condition 2 is used to judge whether all operations that need to be moved forward have finished their left move. The algorithm is stopped if all the last operations on every machine are finished before the 
starting time of the $2 x-1$ th period. Otherwise, the algorithm checks if there is any forwarded operation in the $2 x-1$ th period. If there is none, the algorithm goes to $2(x+1)-1$ th period (the next GAP) to do the forwarded operation selection. Otherwise, the algorithm executes the forwarded operation ranking.

Forwarded operations ranking: After all forwarded operations in the $2 x-1$ th period have been found, they need to be ranked to find out the one with the highest priority for forward (left) moving. The ranking rules are: for operations from different jobs, the one with a higher ratio of its importance compared to its due date $\left(w_{i} / d_{i}\right)$ gets the priority for shifting left. This means urgent jobs with higher importance are prioritised. If the values of $w_{i} / d_{i}$ for the two operations are the same, the one with higher value in $w_{i}$ is prioritised. When $w_{i}$ and $d_{i}$ of the two operations are the same, they are randomly ranked. For operations from the same job, the one positioned earlier in the technology path is prioritised.

Forwarded operation left moving: The different types of forwarded moving can be referred to Figure 5-Figure 7. Suppose $O_{i k}^{l}$ has the highest priority for forward moving in the $2 x-1$ th period, the forward moving rules can refer to the description in the forwarded operations selection step.

\subsection{Comparison of scheduling plans}

As case studies, four modified job shop instances which incorporate electrical consumption profiles for the machine tools have been developed based on the F\&T $10 \times 10$ instance (Fisher \& Thompson, 1963), Lawrence $15 \times 10,20 \times 10$ and $15 \times 15$ job shop instances. The details of the modified job shop instances can be seen in Liu (2014). The due date for each job is defined by the TWK due date assignment method (Shi et al., 2007), $d_{i}=f \times \sum_{i=1}^{m} p_{i k}^{l}, i=1,2, \cdots, n$ where $f$ is the tardiness factor. The value of $f$ is set to1.5, 1.6, 1.7, 1.8 and 1.9. These values correspond to the trend of less tight due dates. For instance, $f=1.5$, represents a tight due date case (corresponds to $50 \%$ tardy jobs). The weight of each $J_{i}$ is randomly allocated. The time unit is defined as minutes. Assuming that all the machine tools in this research are automated ones, the individual value of the idle power level, electricity consumed by Turn off/Turn on operation of each machine and the electricity consumed by processing each operation in the aforementioned four job shops can be abstracted from research works developed by Dahmus (2007) and Drake et al. (2006). Considering the possibility of accelerating machine wear by frequent turn off and turn on operations, the Turn Off/Turn On is only applied when the idle time on the machine is longer than $30 \mathrm{~min}$. The electricity supply pattern is developed based on the fact that in some areas in China, the government electricity is only available 
from Monday to Thursday in every week, which means possibly 3/7 of the production time the private electricity has to be employed. The private electricity nearly doubles the price of the governmental one. Thus, it has been assumed that the electricity price $p^{e}=12.5 \mathrm{MUs} / \mathrm{kWh}$ if it is government electricity supply, while $p^{e}=20.5 \mathrm{MUs} / \mathrm{kWh}$ if it is private electricity supply. The cycle period $T$ of the Rolling Blackout policy is 10 hours, $\Delta t_{s}=480 \mathrm{~min}$ and $\Delta t_{o}=120 \mathrm{~min}$. Based on the experimental results, all job shop instances behave the same. Thus, only the comparison results of E-Lawrence $15 \times 15$ job shop with $f=1.6$ is used as an example to show the trend, as shown in Figure 8. Other experimental results can be referred to Liu (2014). Four solutions form the Pareto front of Lawrence $15 \times 15$ job shop when $f=1.6$. Thus in following figures, the horizontal axis represents the number of solutions.
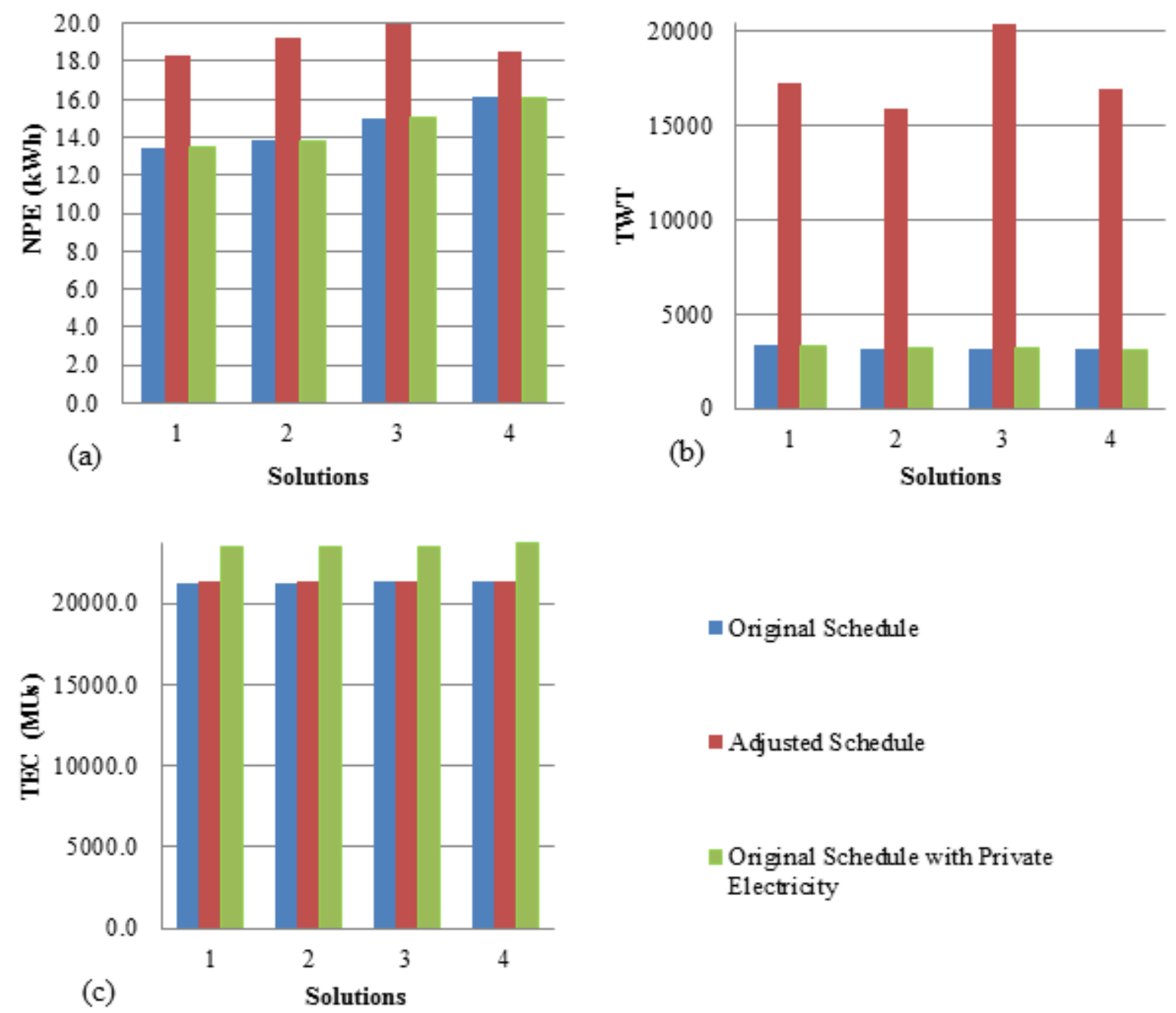

Figure 8 NPE (a), TWT (b) and electricity cost (c) comparisons between the original schedule, adjusted schedule and original schedule with private electricity

Since the private electricity supply is unavailable for the adjusted schedules, it can be observed from part (a) and (b) of Figure 8 that both the total NPE and TWT increased in adjusted schedules after 
postponing some operations in the original schedules. Compared to the original schedules, the total electricity cost in adjusted schedules and original schedules with private electricity consumption are increased. The cost in OSPE is the highest since the private electricity supply is utilised, as shown in part (c) of Figure 8. Based on the above comparison experiments, it can be found that the TWT is sacrificed if the private electricity cannot be used during the blackout periods, while the TEC is sacrificed if the private electricity is used. To deliver the trade-off between TWT and TEC, compromised plans for using the private electricity need to be developed. This leads to the EC2T problem which is solved by a NSGA-II as detailed described below.

\section{Solving the EC2T with NSGA-II}

The goal of this section is to generate schedules which have are a better compromise for using the private electricity. The NSGA-II (Deb et al., 2002) has been adapted to realise the trade-off between the total weighted tardiness and the total electricity cost. New encoding schema, crossover and mutation operators are provided for solving the EC2T problem. They are described in detailed in the following sections. The new method is used to decide whether to provide private electricity to each machine in the job shop during each government electricity supply unavailable period when the Rolling Blackout policy is applied. Then, the operations are scheduled according to the final electricity supply situation.

\subsection{Encoding schema and schedule builder}

The operation-based encoding schema (OBES) and active schedule builder are adopted for this research (Dahal et al., 2007). It can be referred to the authors' previous research (Liu et al., 2014) for more details about them. The chromosome is composed of two parts. The first part is the OBES which is used to represent the priority for operations to be assigned to the machines. The second part of the chromosome, denoted by ESP, is created to represent the private electricity supply plan for each machine during each government electricity supply unavailable period. A typical chromosome for the $3 \times 3$ job shop provided by Liu $\& \mathrm{Wu}(2008)$ is shown below.

$$
[222333111]+\left[\begin{array}{cccccc}
1 & 0 & 0 & 1 & 0 & 1 \\
0 & 1 & 0 & 0 & 1 & 0 \\
0 & 0 & 1 & 0 & 1 & 0
\end{array}\right]
$$

The ESP part of the chromosome is a matrix. The rows represent machines. The columns represent the government electricity supply unavailable periods. For instance, the first row corresponds to machine $M_{1}$, the first column corresponds to the first GUP in the scheduling plan. In ESP, "1" means 
the private electricity supply is available for a given machine during a specific GUP, 0 otherwise. For a specific job shop, the size of the matrix of ESP is decided by the number of machines and the maximum number of GUPs in its schedules after the schedules been adjusted by the above heuristic when the Rolling Blackout policy is applied. Based on some test experiments, it has been found that for a specific job shop, when the number of columns in ESP is set to double the maximum number of GUPs in its adjusted schedules, the algorithm can normally execute successfully. For the above $3 \times 3$ job shop and the OBES part of the chromosome, based on the scheduling adjustment result as shown in Figure 4, 3 GUPs can be identified in the adjusted schedule. Thus, in the ESP part of the above chromosome, the number of rows is 3 and the number of columns is 6 . In the schedule building process, the operation under treatment is allocated to the best available processing time on the required machine. If any of the processing times of an operation overlaps with a GUP, the operation is moved to the earliest available GAP, unless the private electricity supply is available for that GUP. For instance, the corresponding scheduling plan for the above chromosome is shown in Figure 9.

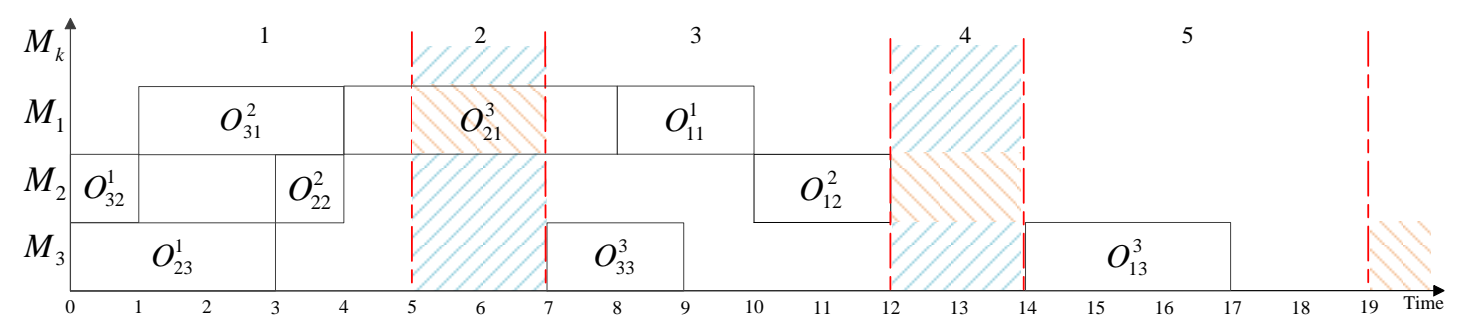

Figure 9 A typical compromised scheduling plan for using private electricity

\subsection{Crossover and mutation operators}

The operation-based order crossover operator is employed for the OBES part of the chromosome (Liu et al., 2014). For the ESP part, the one point crossover operator is adopted. Given parent $A_{1}$ and parent $A_{2}$, the one point crossover operator generates child $A_{1}^{\prime}$ and child $A_{2}^{\prime}$ by randomly choose the same crossover point from both of the parents, and then exchange all the genes before the crossover point in $A_{1}$ and $A_{2}$.

For example, in a $3 \times 3$ job shop, $A_{1}$ and $A_{2}$ are shown as below, $\vdots$ represents the crossover point.

$$
\begin{aligned}
& A_{1}=\left[\begin{array}{lllllll}
1 & 0 & \vdots & 0 & 1 & 1 & 1 \\
0 & 1 & 0 & 0 & 1 & 0 \\
1 & 1 \vdots & 1 & 0 & 1 & 0
\end{array}\right] \\
& A_{2}=\left[\begin{array}{lllllll}
0 & 0 & \vdots & 0 & 1 & 0 & 1 \\
1 & 1 \vdots & 0 & 0 & 1 & 0 \\
1 & 0 & \vdots & 0 & 0 & 1 & 0
\end{array}\right]
\end{aligned}
$$


$A_{1}^{\prime}$ and $A_{2}^{\prime}$ are feasible child chromosomes as shown below.

$$
\begin{aligned}
& A_{1}^{\prime}=\left[\begin{array}{lllllll}
0 & 0 & \vdots & 0 & 1 & 1 & 1 \\
1 & 1 \vdots & 0 & 0 & 1 & 0 \\
1 & 0 \vdots & 1 & 0 & 1 & 0
\end{array}\right] \\
& A_{2}^{\prime}=\left[\begin{array}{llllll}
1 & 0 \vdots & 0 & 1 & 0 & 1 \\
0 & 1 \vdots & 0 & 0 & 1 & 0 \\
1 & 1 \vdots & 0 & 0 & 1 & 0
\end{array}\right]
\end{aligned}
$$

The latter half of all the columns in the ESP are just spare GUPs for schedule building, they will not influence the scheduling result. Thus, the crossover point is always located in the first half of the columns.

Also, the one point mutation operator is employed. Namely, an arbitrary gene in each row of the parent chromosome is chosen and its value is switched. Following the above example, $A_{1}^{\prime \prime}$ is the final child chromosome of $A_{1}$ after applying mutation on $A_{1}^{\prime}$.

$$
\begin{gathered}
A_{1}^{\prime}=\left[\begin{array}{cccccc}
0 & 0 & 0 & 1 & 1 & 1 \\
1 & 1 & 0 & 0 & 1 & 0 \\
1 & 0 & 1 & 0 & 1 & 0
\end{array}\right] \\
A_{1}^{\prime \prime}=\left[\begin{array}{lllllll}
1 & 0 & 0 & 1 & 1 & 1 \\
1 & 0 & 0 & 0 & 1 & 0 \\
1 & 0 & 0 & 0 & 1 & 0
\end{array}\right]
\end{gathered}
$$

The selection operator is a binary tournament (Deb et al., 2002). The maximum number of generations is used as the stopping criterion.

\subsection{Comparison experiment}

To validate the effectiveness of NSGA-II in solving the EC2T problem, a case study has been analysed in this section. The scheduling plans delivered by NSGA-II have been compared with the adjusted schedules and original schedules with private electricity. The four modified job shop instances mentioned in Section 4.2 have been used as the test cases. The parameter settings in the NSGA-II were obtained after the initial tuning process. For all job shop instances, the values are as follows: population size $N=500$; crossover probability $p_{c}=0.9$; mutation probability $p_{m}=0.2$; generation $t=40,000$. The electricity supply pattern is the same as the one which has been described in Section 4.2. The machines are turn off if the idle period is longer than $30 \mathrm{~min}$. Based on the experiment results, the changing trend of the objective function values are the same for all the aforementioned 
job shop instances. Thus, only the E-Lawrence $15 \times 10$ job shop with $f=1.6$ is used as the example to show the comparison. Other experimental results can be referred to Liu (2014). The comparison results are shown in Figure 10, Figure 11 and Figure 12.

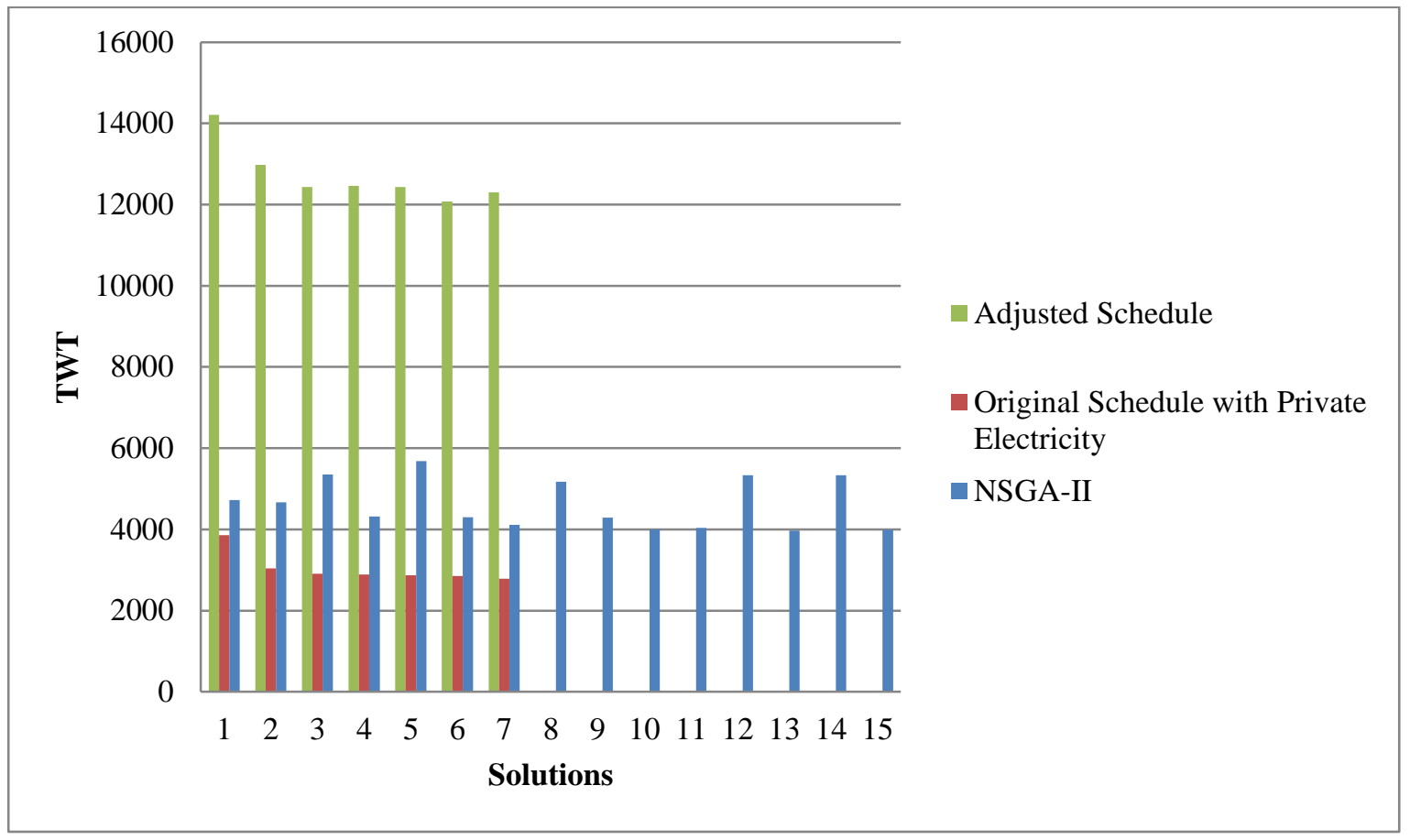

Figure 10 TWT comparison between adjusted schedules, original schedules with private electricity and schedules delivered by NSGA-II

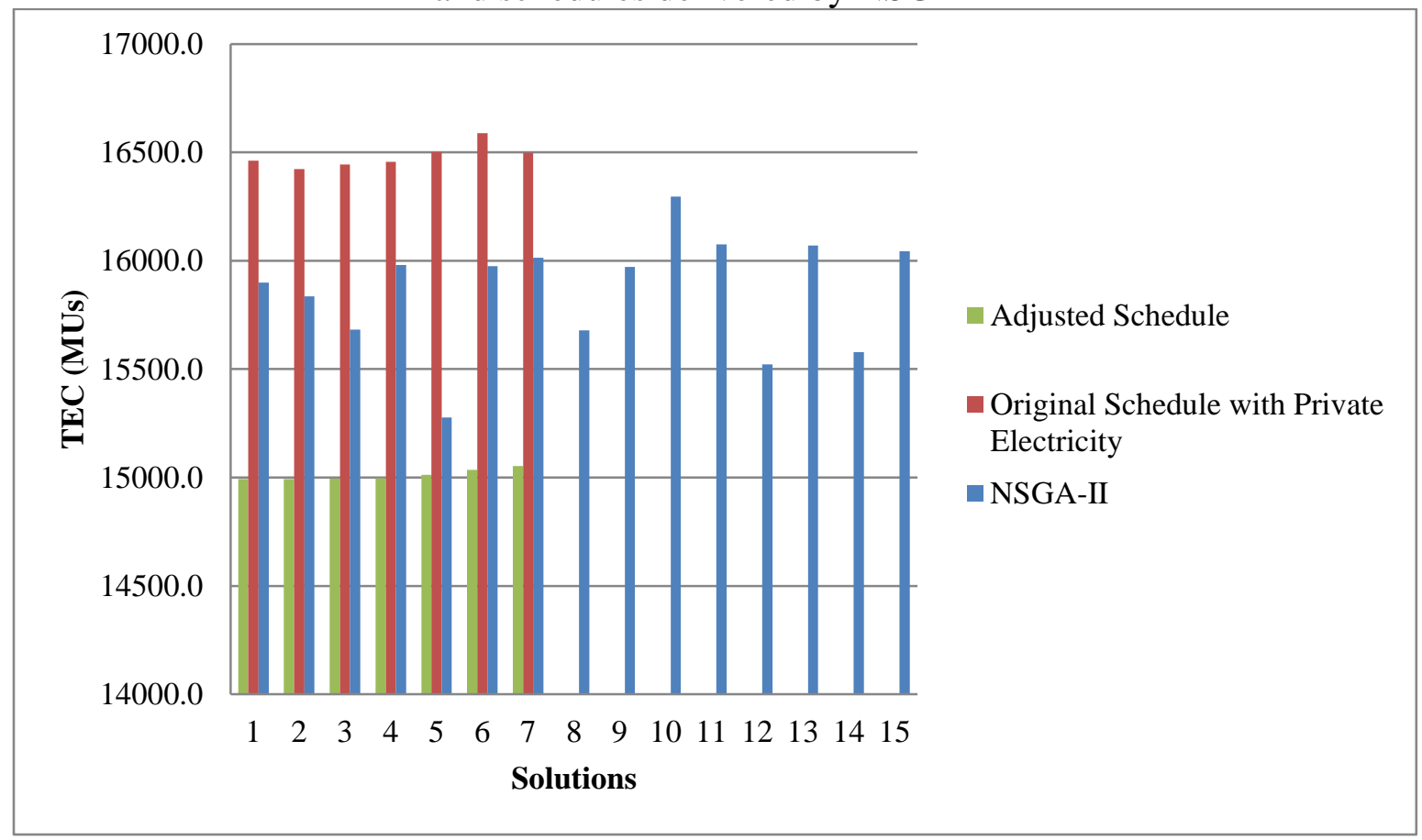

Figure 11 TEC comparison between adjusted schedules, original schedules with private electricity and schedules delivered by NSGA-II 


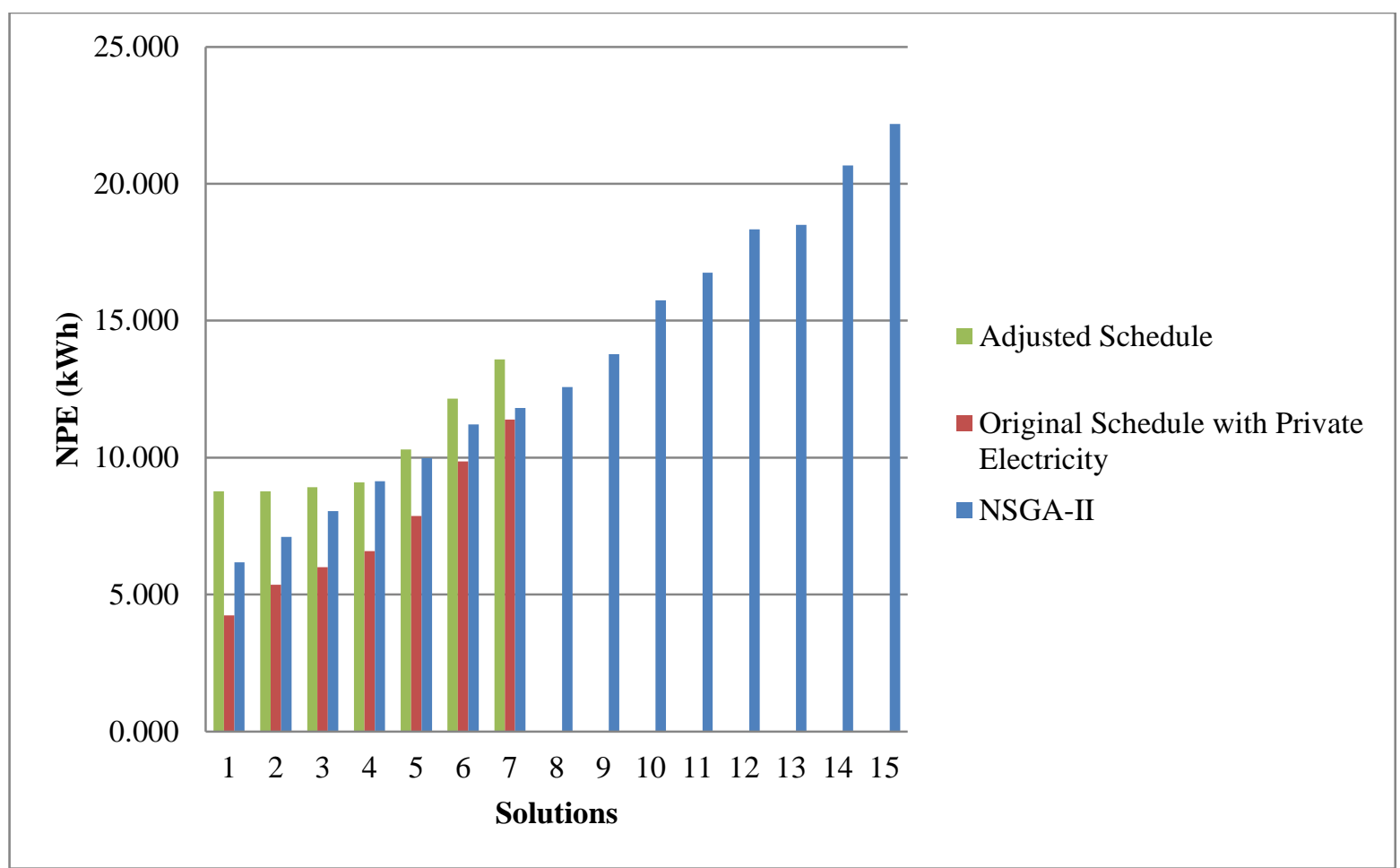

Figure 12 NPE comparison between adjusted schedules, original schedules with private electricity and schedules delivered by NSGA-II

Table 1 The average TWT, TEC and NPE values for adjusted schedules, original schedules with private electricity and schedules delivered by NSGA-II

\begin{tabular}{llll}
\hline & Average TWT (weighted minute) & Average TEC (MUs) & Average NPE (kWh) \\
\hline AS & 12699.57 & 15018.2 & 10.228 \\
\hline OSPE & 3028.571 & 16482.3 & 7.3 \\
\hline NSGA-II & 4617.667 & 15860.0 & 13.0 \\
\hline
\end{tabular}

According to Figure 10 and Table 1, the scheduling plans obtained by NSGA-II have better total weighted tardiness performance compared to the adjusted schedules. For the NSGA-II solutions, the average value in TWT is 4617.7 weighted minutes while in the adjusted scenario it is 12699.6 weighted minutes. The minimum improvement is $61.65 \%$, the maximum improvement is $71.95 \%$ and the average improvement is $63.64 \%$. According to Figure 11, the scheduling plans obtained by NSGA-II have better total electricity cost performance compared to the original schedules with private electricity. For the NSGA-II solutions, the average value in TEC is 15860.0 money units while in the private electricity usage scenario it is 16482.3 money units. The minimum improvement is $0.78 \%$, the maximum improvement is $7.91 \%$ and the average improvement is $3.78 \%$. According to Table 1, the average value of the total non-processing electricity is slightly increased in NSGA-II solutions compared to the other two scenarios. However, it can be observed from Figure 12 that some of the NSGA-II solutions outperform the adjusted schedules in terms of NPE. Thus, it can be 
concluded that the compromised plans for using private electricity developed by NSGA-II, is effective to realise the tri-objective optimisation to minimise total electricity cost, total non-processing electricity consumption and total weighted tardiness in job shops when the Rolling Blackout policy is applied. Based on the experiment results, it can be found that the heuristic developed in this research can help the plant managers to understand the potential loss of their factories when the Rolling Blackout policy is applied in terms of total weighted tardiness or electricity cost. On the other hand, this heuristic can also help the plant manager to reduce the loss in delivery. More significantly, based on the understanding of the influence of the Rolling Blackout policy, this research has developed an approach based on NSGA-II which can help the plant manager to optimally use the private electricity to achieve cost reduction and better performance on delivery. Based on the experiment result shown in Figure 11, in the scenario of Original Schedule with Private Electricity, the electricity used to accomplish a specific schedule has a proportion of private electricity as $15.63 \%$. While this value decreased to $10.42 \%$ in the NSGA-II scenario. This means the cost of electricity can be saved. On the other hand, the private electricity nearly generates double the amount of emissions compared to public electricity. Considering this, it can be estimated that the $\mathrm{CO}_{2}$ emissions resulting from an optimised schedule can be reduced by approximately $4.4 \%$ compared to a schedule which has not be optimised considering the impact of Rolling Blackouts and usage of private electricity. Hence, the proposed scheduling method can help manufacturing companies to both improve their cost as well as reduce their emission, thereby, benefiting the environment.

\section{Conclusions and Future Work}

The Rolling Blackout policy implemented in China deteriorates the performance of existing scheduling plans of manufacturing companies. To overcome this, some companies start their own diesel generator to maintain the production, which significantly increased their electricity costs and also has an adverse effect on the environment due to higher $\mathrm{CO}_{2}$ emissions. Thus, reducing electricity consumption and its related cost as well as keeping good performance in classical scheduling objectives in job shops is a difficult problem that can take a large amount of time to optimally solve. The mathematical model is formalised for the tri-objective job shop scheduling problem that consider minimising total electricity cost, total non-processing electricity consumption and total weighted tardiness when the Rolling Blackout policy is applied. To understand the influence that the Rolling Blackout policy exerts on the existing scheduling plans, a heuristic has been developed to adjust existing scheduling plans to fit the government electricity supply time frame. Three types of schedule, including the original schedules, the adjusted schedules and original schedules with private electricity have 
been solved for four modified job shop instances. They have been compared with each other to demonstrate that either the total weighted tardiness and the total electricity cost need to be compromised independently of whether private electricity is used or not during government electricity unavailable periods. The E-Lawrence $15 \times 15$ job shop with the tardiness factor of 1.6 was used as an example to show the aforementioned sacrifice. There is a requirement to realise the trade-off between total weighted tardiness and total electricity cost. Thus, the NSGA-II is adapted to solve the EC2T problem. Based on the case study and comparison experiments, it has been proved that the algorithm is effective in reducing total weighted tardiness and total electricity cost compare to the adjusted schedules and original schedules with private electricity, respectively.

The environmental impact of production during Rolling Blackouts can also be reduced by achieve good scheduling performance which requires less diesel generated electricity. The significance of improved scheduling performance can be demonstrated using the Whenzhou area of Zhejiang Province in China as an example. There are more than 300,000 SMEs in in this province and the Rolling Blackout policy has been strictly applied for these companies during summer time in recent years. The SMEs mentioned above are operated under similar conditions as those used in the 4 test cases. According to the experiment results, the delivery can be improved between $60 \%$ and $70 \%$. Conservatively, this indicates typical SMEs operating in this area will be able to improve the tardiness from Rolling Blackouts by at least 50\% if the optimisation method developed in this research is applied. Simultaneously, their cost for using privately generated electricity can also be reduced by avoiding high energy usage processes during Blackout periods. More importantly, this will reduce the emission caused by local electricity generation. Considering the private electricity generates double amount of emissions to the public electricity, which means approximately $4.4 \%$ of the $\mathrm{CO}_{2}$ emission reduction can be achieved. These results demonstrate the importance of considering energy availability and different generation methods during scheduling.

The clear advantage of the developed method is that it can help identify both potential for cost saving as well as reducing environmental impact without any significant additional investment other than for the mathematical tools proposed in this paper. In future work, the developed heuristic and the NSGA-II should be tested on wider set of job shop scenarios to validate its more general applicability and potential for reducing environmental impact of Rolling Blackouts. 


\section{Reference}

Bruzzonea, A.A.G., Anghinolfib, D., Paoluccib, M., Tonellia, F., 2012. Energy-aware scheduling for improving manufacturing process sustainability: A mathematical model for flexible flow shops. CIRP Annals - Manufacturing Technology 61, 459-462.

Dahal, K.P., Tan, K.C., Cowling, P.1., 2007. Evolutionary Scheduling.

Dahmus, J.B., 2007. Applications of Industrial Ecology Manufacturing, Recycling, and Efficiency, Department of Mechanical Engineering. Massachusetts Institute of Technology.

Deb, K., Pratap, A., Agarwal, S., Meyarivan, T., 2002. A Fast and Elitist Multiobjective Genetic Algorithm NSGA-II. IEEE Transactions on Evolutionary Computation 6, 182-197.

Drake, R., Yildirim, M.B., Twomey, J., Whitman, L., Ahmad, J., Lodhia, P., 2006. Data collection framework on energy consumption in manufacturing, Institute of Industrial Engineering Research Conference.

Fang, K., Uhan, N., Zhao, F., Sutherland, J.W., 2011. A New Shop Scheduling Approach in Support of Sustainable Manufacturing, Berlin, Heidelberg, pp. 305-310.

Fisher, H., Thompson, G.L., 1963. Probabilistic Learning Combinations of Local Job-Shop Scheduling Rules, in: Thompson, J.F.M.a.G.L. (Ed.), Industrial Scheduling. Prentice-Hall, Englewood Cliffs, NJ, pp. 225-251.

Gutowski, T., Murphy, C., Allen, D., Bauer, D., Bras, B., Piwonka, T., Sheng, P., Sutherland, J., Thurston, D., Wolff, E., 2005. Environmentally benign manufacturing: Observations from Japan, Europe and the United States. Journal of Cleaner Production 13, 1-17.

He, Y., Liu, B., Zhang, X., Gao, H., Liu, X., 2012. A modeling method of task-oriented energy consumption for machining manufacturing system. Journal of Cleaner Production 23, 167-174.

Kilian, L., 2008. The Economic Effects of Energy Price Shocks. Journal of Economic Literature 46, 871-909.

Kordonowy, D., 2003. A power assessment of machining tools. Massachusetts Institue of Technology.

Liu, M., Wu, C., 2008. Intelligent Optimization Scheduling Algorithms for Manufacturing Process and Their Applications. National Defense Industry Press.

Liu, Y., 2014. Multi-objective Optimisation Methods for Min-imising Total Weighted Tardiness, Electricity Consumption and Electricity Cost in Job Shops Through Scheduling. University of Nottingham.

Liu, Y., Dong, H., Lohse, N., Petrovic, S., Gindy, N., 2014. An investigation into minimising total energy consumption and total weighted tardiness in job shops. Journal of Cleaner Production 65, 87-96. 
Mouzon, G., 2008. Operational methods and models for minimisation of energy consumption in a manufacturing environment. Wichita State University.

Mouzon, G., Yildirim, M.B., 2008. A framework to minimize total energy consumption and total tardiness on a single machine, Wichita State University, pp. 105-116.

Mouzon, G., Yildirim, M.B., Twomey, J., 2007. Operational methods for minimization of energy consumption of manufacturing equipment. International Journal of Production Research 45, 42474271.

Özgüven, C., Özbakır, L., Yavuz, Y., 2010. Mathematical models for job-shop scheduling problems with routing and process plan flexibility. Applied Mathematical Modelling 34, 1539-1548.

Shi, R., Zhou, Y., Zhou, H., 2007. A hybrid evolutionary algorithm for bi-objective job shop scheduling problems. Control and Decision 22, 1228-1234.

Subaï, C., Baptiste, P., Niel, E., 2006. Scheduling issues for environmentally responsible manufacturing: The case of hoist scheduling in an electroplating line. International Journal of Production Economics 99, 74-87.

Tang, D., Du, K., Li, L., 2006. On the development path of Chinese manufacturing industry based on resource restraint. Jiangsu Social Sciences 4, 51-58. 